\title{
Análisis de la atención en salud de los pacientes diagnosticados con mieloma múltiple en Colombia
}

\section{Analysis of health care of patients diagnosed with multiple myeloma in Colombia}

\author{
Yolima Méndez Camacho', Natalia Arias Amézquita², Mónica Giraldo Castaño³, Diana Valenzuela Cadena ${ }^{4}$
}

1 Psicóloga. Presidenta, Fundación Colombiana de Leucemia y Linfoma.

Trabajadora social, magíster en Salud Pública. Fundación Colombiana de Leucemia y Linfoma.

Psicóloga, magíster en Psicología Clínica. Fundación Colombiana de Leucemia y Linfoma.

Administradora pública. Fundación Colombiana de Leucemia y Linfoma.

\section{Resumen}

Se realizó investigación no experimental (mixta) transversal, de tipo descriptivo. Se procesó información de 127 pacientes con mieloma múltiple (MM) en Colombia que reciben tratamiento en ocho ciudades del país.

Objetivo: identificar el proceso de atención en los pacientes con MM y las características de oportunidad y calidad en diagnóstico y tratamiento.

Metodología: se aplicó un cuestionario estructurado, reseñando según respuestas los tiempos de atención desde el inicio de síntomas hasta su tratamiento actual, pasando por los diagnósticos erróneos en los que se incurrió. En complemento, se realizaron cuatro grupos focales en las ciudades de Bogotá, Medellín, Cali y Barranquilla. Para el análisis de los resultados, se utilizó el marco normativo del Sistema Obligatorio de Garantía de Calidad (SOGC) del país y estudios relacionados.

Resultados: un $68 \%$ de pacientes con MM refirió haber recibido diagnósticos erróneos antes de su diagnóstico definitivo. Un 35\% recurrió a acción de tutela para el acceso a tratamiento. Un $27 \%$ no inició tratamiento con oportunidad. El $46 \%$ de los pacientes fue remitido a hematología en un tiempo máximo de 1 mes; el 24\%, entre 2 y 4 meses; el $24 \%$, entre 5 meses y un año; y el $6 \%$, más de 1 año.

Conclusiones: se identificó falta de oportunidad en la detección efectiva del MM. Una vez diagnosticados, se observó oportunidad en el acceso al tratamiento oncológico. Se expone la necesidad de visibilizar y sensibilizar a pacientes, personal asistencial y población en general a favor de la detección eficaz y oportuna del MM.

Palabras clave: mieloma múltiple, prestación de atención de salud, accesibilidad a los servicios de salud, Colombia, salud pública. (Fuente: DeCS).

\begin{abstract}
Non-experimental research (mixed) cross-sectional, of descriptive type information was processed of 127 patients with multiple myeloma (MM) in Colombia, who receive treatment in 8 cities of the country.

Objective: To identify the health-care process of patients with MM, and the characteristics of opportunity and quality in diagnosis and treatment.

Methodology: A structured questionnaire is made describing according to the answers, the times of attention from the beginning of symptoms to its current treatment, through the misdiagnosis in which it was incurred. In addition, 4 focus groups are held in the cities of Bogotá, Medellín, Cali and Barranquilla. For the analysis of the results we used the regulatory framework of the Mandatory Quality Assurance System in Colombia (SOGC).

Results: $68 \%$ patients reported having received misdiagnosis prior the final diagnosis. $35 \%$ used the writ of injunction to claim their health rights. $27 \%$ didn't start timely treatment. $46 \%$ of the patients were referred to the hematologist within onemonth maximum, 24\% between 2 and 4 months, 24\% between 5 months and 1 year, and $6 \%$ mare that 1 year.

Conclusions: It was identified a lack of opportunity in the diagnosis of the MM. Once the diagnosis is confirmed, it was evidenced opportunity in the access to the treatment. It is important highlight the need of awareness actions to patients and care personal, in pro of an opportunity and effective diagnosis of MM.
\end{abstract}

Key words: Multiple myeloma, public health, health service accessibility, healthcare provision, Colombia. (Source: MeSH). 


\section{Introducción}

Según cifras del Instituto Nacional de Cancerología', en el año 2013 se presentaron 56 nuevos casos de MM en Colombia (24 hombres-32 mujeres), correspondiendo a un $19,5 \%$ de enfermedades en el sistema hematopoyético. Para este mismo año, la mortalidad por MM es del 1,0\%. De acuerdo con datos de Globocan $^{2}$, se estima que cerca de 600 personas en Colombia padecerían mieloma múltiple en los próximos años, con un riesgo acumulado de 0,17. La baja incidencia de este diagnóstico en el país referencia la falta de información sobre las características y necesidades de atención en salud de esta población en Colombia. Conforme con revisión bibliográfica, no se evidencian estudios en nuestro país en los que se pueda obtener información de estos pacientes y sus necesidades en particular. Se encontraron artículos relacionados con el impacto de la tardanza (delay) en el diagnóstico de MM en el Reino Unido ${ }^{3}$. Surge entonces el interés por parte de la Fundación Colombiana de Leucemia y Linfoma en la generación de información que aporte a los profesionales de la salud en su comprensión de la atención a los pacientes con MM en el país, brindando herramientas para intervenciones efectivas. La información aquí procesada arroja datos importantes para la construcción de un diagnóstico situacional en el que organizaciones sociales y de salud interesadas en la atención y abordaje del mieloma múltiple puedan brindar una mejor atención a los pacientes y sus familias.

\section{Material y métodos}

Se llevó a cabo investigación no experimental (mixta) transversal de tipo descriptivo por parte de la Fundación Colombiana de Leucemia y Linfoma en el año 2017. Para el presente artículo, se privilegian los resultados del análisis cuantitativo realizado.

Los participantes del estudio fueron pacientes adscritos a la Fundación que, a la fecha, reciben atención y asesoría para la superación de barreras de acceso a los servicios de salud que requieren. La selección de esta población correspondió a un tipo de muestreo no probabilístico basado en la muestra de sujetos voluntarios (muestreo intencional por cumplimiento de características del perfil de interés, único criterio de inclusión: diagnóstico de MM). La recolección de información se llevó a cabo mediante encuesta telefónica a 127 pacientes, con cuestionario estructurado basado en formularios de encuestas realizadas por la Asociación Española de Afectados por Linfoma, Mieloma y Leucemia $(A E A L)^{9}$ y la Asociación Brasileña de Linfoma y Leucemia (Abrale). Se hicieron los ajustes correspondientes al cuestionario para recopilar información sobre los tiempos de atención que gastan estos pacientes dentro del sistema de salud colombiano. En complemento, se llevaron a cabo cuatro grupos focales en las ciudades de Bogotá, D.C., Cali, Barranquilla y Medellín, con el fin de identificar narrativas sobre el proceso de diagnóstico/tratamiento y sus necesidades particulares por región.

La información de tipo cuantitativo se procesó a partir de la identificación de la proporción de pacientes con diagnósticos erróneos y barreras de acceso, al igual que la proporción y frecuencias de tiempos de acceso a los servicios de salud requeridos por los pacientes. Estos elementos fueron analizados para describir una 'línea de tiempo' que buscaba identificar los períodos entre los síntomas, la atención por médico general, el médico especialista, el diagnóstico y el tratamiento efectivo del MM (ver figura 1), a partir de la siguiente información recabada: 1) fecha de diagnóstico, 2) tiempo entre síntomas y consulta al médico, 3) tiempo entre primera visita y diagnóstico final, 4) tiempo entre primera visita y remisión a especialista, 5) tiempo entre diagnóstico y tratamiento.

Los criterios de análisis para la identificación de calidad de la atención fueron: 1) diagnóstico erróneo, 2) cambio de IPS por decisión de la EPS, 3) negación de servicios, 4) fragmentación de la atención, 5) acciones jurídicas implementadas.

Para el análisis de los criterios de oportunidad y calidad, se utilizaron el marco normativo del SOGC4,

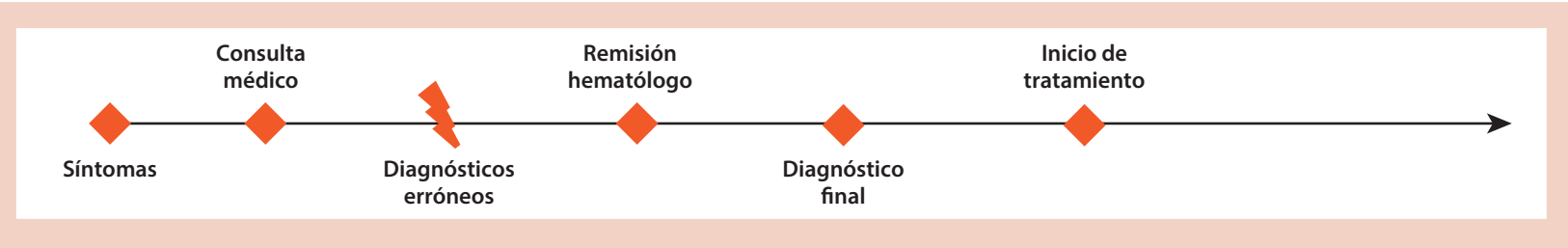

Figura 1. Línea de tiempo y hechos de análisis. 
Tabla 1. Actividad ocupacional, nivel educativo, aseguramiento en salud y estrato socioeconómico en pacientes con MM participantes del estudio

\begin{tabular}{|c|c|c|c|c|c|c|c|c|c|c|c|}
\hline $\begin{array}{l}\text { Actividad } \\
\text { ocupacional }\end{array}$ & $\#$ & $\%$ & Nivel educativo & $\#$ & $\%$ & $\begin{array}{c}\text { Aseguramiento } \\
\text { en salud }\end{array}$ & $\#$ & $\%$ & $\begin{array}{l}\text { Estrato } \\
\text { socioeconómico }\end{array}$ & $\#$ & $\%$ \\
\hline Pensionado & 38 & $30 \%$ & Bachiller & 21 & $16,5 \%$ & Régimen contributivo & 85 & $67 \%$ & Estratos 1-2 & 73 & $57 \%$ \\
\hline $\begin{array}{l}\text { Incapacitado } \\
\text { por enfermedad }\end{array}$ & 36 & $28 \%$ & Básica primaria & 42 & $33 \%$ & Régimen subsidiado & 31 & $24 \%$ & Estratos 3-4 & 50 & $40 \%$ \\
\hline Oficios del hogar & 22 & $17 \%$ & No escolarizado & 27 & $21,2 \%$ & Régimen especial & 10 & $8 \%$ & Estratos 5-6 & 4 & $3 \%$ \\
\hline $\begin{array}{c}\text { Trabajador } \\
\text { independiente }\end{array}$ & 15 & $12 \%$ & Posgrado/doctorado & 15 & $12 \%$ & No asegurado/vinculado & 1 & $1 \%$ & & & \\
\hline Sin actividad laboral & 6 & $5 \%$ & Profesional & 22 & $17,3 \%$ & & & & & & \\
\hline Empleado & 5 & $4 \%$ & & & & & & & & & \\
\hline $\begin{array}{c}\text { En condición } \\
\text { de discapacidad }\end{array}$ & 5 & $4 \%$ & & & & & & & & & \\
\hline Total & 127 & $100 \%$ & & 127 & $100 \%$ & & 127 & $100 \%$ & & 127 & $100 \%$ \\
\hline
\end{tabular}

las recomendaciones para la atención oncológica del modelo para el control del cáncer en Colombia del Instituto Nacional de Cancerología (INC) ${ }^{5}$, la Resolución 1477 de $2006^{6}$ y los criterios usados en otros estudios relacionados sobre la tardanza (delay) o inoportunidad en el diagnóstico de mieloma múltiple y la supervivencia de los pacientes ${ }^{3}$.

Los aspectos éticos contemplados en la investigación corresponden a los enunciados en la Resolución 8430 de 1993, en especial el artículo 5º en el que se especifica que en toda investigación en la que el ser humano sea sujeto de estudio deberá prevalecer el criterio del respeto a su dignidad y la protección de sus derechos y su bienestar?. La investigación está catalogada según la misma resolución como 'investigación sin riesgo' y contó con la autorización de los participantes diagnosticados con MM y sus familiares y/o cuidadores, quienes manifestaron su voluntad mediante la firma del consentimiento informado en los grupos focales, y su consentimiento verbal a través de contacto telefónico en la aplicación de las encuestas.

\section{Resultados}

Participaron en el estudio un total de 127 pacientes con MM. De estos, 27 fueron parte de los grupos focales. La distribución por sexo corresponde a 67 mujeres y 60 hombres. El 3\% (4 casos) corresponde a menores de 45 años; el $43 \%$ (55), a personas entre los 46 a 60 años; y un 54\% (68), a personas mayores de 60 años. La tabla 1 expone características socioeconómicas de la población participante del estudio.

Se indagó a los participantes sobre el tiempo transcurrido entre los primeros síntomas y la consulta al médico general. Los resultados muestran que un $73 \%$ (93) consultó a su médico en menos de tres meses. Sobre esta fase inicial entre síntomas y atención por medicina general, se preguntó si los pacientes habían presentado o no diagnósticos erróneos. Según el procesamiento de la información, se encuentra que un $68 \%$ (86) recibió un diagnóstico erróneo (ver tabla 2). Llama la atención el caso de pacientes diagnosticados con problemas psicológicos (estrés), con problemas dentales y cálculos biliares.

Sobre el tiempo transcurrido entre la primera visita al médico general y la remisión al especialista (hematólogo), se encontró que un $46 \%$ (58) de los pacientes fue remitido en un tiempo máximo de 1 mes; el 24\% (30), entre 2 y 4 meses; el 24\% (31), entre 5 meses y un año; y el 6\% (8), más de 1 año (ver tabla 3).

Se observa que el 49\% (42) de los pacientes del régimen contributivo fueron remitidos a hematología dentro del primer mes de consulta, mientras que en el régimen subsidiado el $39 \%$ (12). Por otro lado, es importante resaltar que el 13\% (4) de los pacientes del régimen subsidiado tardaron más de 1 año en ser remitidos al especialista y el 26\% (8), entre 5 y 7 meses. Llama la atención también que el $40 \%$ (4) de los pacientes del régimen especial fueron remitidos entre el octavo y duodécimo mes posterior a la consulta

Tabla 2. Diagnósticos erróneos emitidos previos al diagnóstico de mieloma múltiple

\begin{tabular}{|l|c|c|}
\hline \multicolumn{1}{|c|}{ Diagnóstico } & $\#$ & $\%$ \\
\hline Afectación muscular & 18 & $20,9 \%$ \\
\hline Afectación ósea & 15 & $17,4 \%$ \\
\hline Enfermedad común del adulto mayor & 13 & $15,1 \%$ \\
\hline Diagnóstico hematológico & 8 & $9,3 \%$ \\
\hline No especifica & 6 & $7,0 \%$ \\
\hline Otro tipo de cáncer & 6 & $7,0 \%$ \\
\hline Afección respiratoria & 4 & $4,7 \%$ \\
\hline Afectación genitourinaria & 4 & $4,7 \%$ \\
\hline Afectación en aparato digestivo & 3 & $3,5 \%$ \\
\hline Estrés & 3 & $3,5 \%$ \\
\hline Problemas cardíacos & 3 & $3,5 \%$ \\
\hline Afectación del sistema circulatorio & 2 & $2,3 \%$ \\
\hline Enfermedad congénita & 1 & $1,1 \%$ \\
\hline Total & $\mathbf{8 6}$ & $\mathbf{1 0 0} \%$ \\
\hline
\end{tabular}


Tabla 3. Tiempos de acceso a consulta por hematología según régimen de afiliación en salud

\begin{tabular}{|c|c|c|c|c|c|c|c|c|c|c|c|c|c|}
\hline \multirow{2}{*}{$\begin{array}{l}\text { Régimen de afiliación } \\
\text { Régimen contributivo }\end{array}$} & \multicolumn{2}{|c|}{$\begin{array}{l}\text { Entre 1-2 } \\
\text { semanas }\end{array}$} & \multicolumn{2}{|c|}{$\begin{array}{l}\text { Entre 2-4 } \\
\text { semanas }\end{array}$} & \multicolumn{2}{|c|}{$\begin{array}{c}\text { Entre } \\
\text { 2-4 meses }\end{array}$} & \multicolumn{2}{|c|}{$\begin{array}{l}\text { Entre } \\
\text { 5-7 meses }\end{array}$} & \multicolumn{2}{|c|}{$\begin{array}{c}\text { Entre 8-12 } \\
\text { meses }\end{array}$} & \multicolumn{2}{|c|}{$\begin{array}{c}\text { Más de } \\
1 \text { año }\end{array}$} & \multirow{2}{*}{$\begin{array}{r}\text { Total } \\
85\end{array}$} \\
\hline & 23 & $27 \%$ & 19 & $22 \%$ & 23 & $27 \%$ & 9 & $11 \%$ & 7 & $8 \%$ & 4 & $5 \%$ & \\
\hline Régimen subsidiado & 7 & $23 \%$ & 5 & $16 \%$ & 5 & $16 \%$ & 8 & $26 \%$ & 2 & $6 \%$ & 4 & $13 \%$ & 31 \\
\hline Régimen especial & 4 & $40 \%$ & 0 & $0 \%$ & 1 & $10 \%$ & 1 & $10 \%$ & 4 & $40 \%$ & 0 & $0 \%$ & 10 \\
\hline Vinculado & 0 & $0 \%$ & 0 & $0 \%$ & 1 & $100 \%$ & 0 & $0 \%$ & 0 & $0 \%$ & 0 & $0 \%$ & 1 \\
\hline Total & 34 & & 24 & & 30 & & 18 & & 13 & & 8 & & 127 \\
\hline
\end{tabular}

Nota: los porcentajes registrados corresponden al universo de encuestados según régimen de salud.

inicial. Se presentó un caso sin afiliación (vinculado), quien por problemas en su aseguramiento presentó barreras de acceso a su tratamiento, desencadenando su fallecimiento.

La mayoría de pacientes afirmó que el tiempo transcurrido entre la atención suministrada por el médico general y el diagnóstico final osciló entre 2 y 4 meses.

Posterior al diagnóstico, se indagó sobre el tiempo transcurrido entre este y el acceso al tratamiento oncológico, hallando que el 43\% (55) accedió inmediatamente. De la población restante, un 30\% (38) señaló un tiempo entre 1 y 2 semanas para el inicio. Llama la atención que un 4\% (5) refirió demora por más de 5 meses para el inicio del tratamiento.

Para la identificación de barreras de acceso a la atención especializada por hematología, se interrogó a los participantes sobre los tiempos transcurridos para acceder a este servicio según última orden médica recibida. Al respecto, la mayoría de los casos accedió en un rango de 4 semanas.

Se preguntó sobre si estos habían tenido cambio de su IPS por decisión de su EPS (generalmente por cambios en la contratación). Al respecto, se encontró que, de la población participante, al 30,7\% (39) se le cambió de IPS por decisión de la EPS y un 67,7\% (88) indicó no haber presentado este tipo de cambios en su red prestadora de servicios de salud. Sobre la negación de servicios de salud, la mayoría de pacientes encuestados reportó no presentar esta problemática $(83,4 \%, 107)$, un total del $15,7 \%$ (20) refirió negación de servicios, en especial en imaginología y medicamentos.

En la tabla 4, se describen las barreras de acceso según el régimen de salud. El 55\% (17) de los pacientes del régimen subsidiado reportó haber tenido por lo menos una barrera de acceso al sistema de salud. Para el régimen contributivo, un $49 \%$ (42), y un $90 \%$ (9) para el régimen especial.
Tabla 4. Barreras de acceso que reportan los pacientes con mieloma múltiple según régimen de afiliación

\begin{tabular}{|c|c|c|c|c|c|c|c|c|}
\hline \multirow{3}{*}{$\begin{array}{l}\text { Régimen } \\
\text { de salud }\end{array}$} & \multirow{2}{*}{\multicolumn{2}{|c|}{$\begin{array}{c}\begin{array}{c}\text { Cambio } \\
\text { de IPS }\end{array} \\
\text { Sí }\end{array}$}} & \multirow{2}{*}{\multicolumn{2}{|c|}{$\begin{array}{c}\text { Cobro } \\
\text { copago }\end{array}$}} & \multirow{2}{*}{\multicolumn{2}{|c|}{$\begin{array}{c}\text { Negación } \\
\text { de proce- } \\
\text { dimiento } \\
\text { Sí }\end{array}$}} & \multirow{2}{*}{\multicolumn{2}{|c|}{ Total }} \\
\hline & & & & & & & & \\
\hline & $\#$ & $\%$ & $\#$ & $\%$ & $\#$ & $\%$ & $\#$ & $\%$ \\
\hline R. contributivo & 21 & $25 \%$ & 27 & $32 \%$ & 9 & $11 \%$ & 42 & $49 \%$ \\
\hline R. subsidiado & 9 & $29 \%$ & 7 & $23 \%$ & 6 & $19 \%$ & 9 & $90 \%$ \\
\hline R. especial & 9 & $90 \%$ & 0 & $0 \%$ & 4 & $40 \%$ & 17 & $55 \%$ \\
\hline Vinculado/no afiliado & 0 & $0 \%$ & 0 & $0 \%$ & 1 & $100 \%$ & 1 & $100 \%$ \\
\hline
\end{tabular}

En referencia a las acciones legales interpuestas por los pacientes para el acceso a su atención en salud, un 56\% (72) acudió a una acción legal, correspondiendo a un 21\% (27) el derecho de petición y a un 35\% (45) la acción de tutela.

Los testimonios y experiencias recopilados en los grupos focales evidenciaron las dificultades en el diagnóstico y acceso a la atención oncológica que han requerido. Particularmente, los relatos de los pacientes se centraron en las gestiones administrativas que deben realizar ante su seguro de salud (EPS) para la autorización de órdenes médicas y lo que les implica esta demora en su situación de salud y en su gasto promedio mensual.

En los grupos focales de Medellín y Cali, los pacientes residentes en zonas rurales describieron el tiempo por trayecto entre el lugar de residencia y la IPS. Se encontraron seis casos en los que deben trasladarse en promedio ocho horas por trayecto (pacientes de los departamentos de Cauca y Antioquia) hasta llegar a las cabeceras municipales. El promedio de gasto mensual en transporte en las ciudades del país donde se realizaron los grupos focales es de 300.000 pesos (100 dólares), esto sin considerar el gasto de bolsillo para el paciente y su cuidador en temas como alimentación y aseo personal.

\section{Discusión}

Los conceptos de oportunidad y calidad en salud contemplados en el SOGC se centran en servicios accesibles y equitativos para todos los colombianos, 
a través de un nivel profesional óptimo, teniendo en cuenta el balance entre beneficios, riesgos y costos, con el propósito de lograr la adhesión y satisfacción de dichos usuarios ${ }^{4}$. Sobre el tiempo que implicó a los pacientes diagnosticados con MM acceder a su diagnóstico, llama la atención que un $68 \%$ recibiera diagnósticos erróneos relacionados con afectaciones musculares y óseas propias del adulto mayor; es así como los criterios de calidad en la detección acertada y oportuna del diagnóstico se vieron afectados en dichos pacientes.

Si bien este estudio no hizo énfasis en la identificación de la supervivencia de los participantes ante la inoportunidad del diagnóstico, la revisión de otros estudios similares ${ }^{3,8}$ expresa que una tardanza en el diagnóstico de MM por más de seis meses registraba un número mayor de complicaciones en los pacientes. Por lo anterior, se hace necesario el fortalecimiento de campañas de sensibilización y toma de conciencia dirigidas a la comunidad en general y a los futuros médicos en particular, que favorezcan un mayor conocimiento de los síntomas propios del MM. Una mayor agudeza clínica por parte de los médicos de primer nivel de atención dentro del proceso de detección facilitaría la identificación de casos y la remisión oportuna al especialista hematólogo. También es importante el fortalecimiento de campañas dirigidas a la población adulta como estrategia de educación frente a los síntomas de las enfermedades hematooncológicas a partir de la consulta oportuna.

En relación con la atención especializada, un 46\% (58) de los pacientes fue remitido a hematología en un tiempo máximo de 1 mes; un 24\%, entre 2 y 4 meses; un 24\% (30), entre 5 meses y 1 año; y un $6 \%$ (8), más de 1 año para la atención requerida, por lo tanto, un 30\% (39) de los pacientes no contó con la posibilidad de acceder a atención especializada de hematología en el momento requerido, posterior a su remisión desde medicina general. Según las características propias de la atención del MM, este tiempo podría considerarse perjudicial para la atención oportuna del diagnóstico, en el que se esperaría que oscilara entre un mes o menos, de acuerdo con criterios de atención oncológica ${ }^{6}$.

Sobre el acceso a tratamiento, un 73\% (93) expresa haberlo recibido en menos de dos semanas, lo que refleja un estimado sobre los tiempos oportunos de acceso, y, en este sentido, una demora superior a dos semanas puede sugerir falta de oportunidad en la atención del MM.

Que el 35\% (44) de los pacientes del estudio haya tenido que recurrir a una acción de tutela para acceder a una atención en salud sugiere que existe un alto riesgo de vulneración a sus derechos, lo cual reafirma la importancia de hacer seguimiento continuo a los pacientes, así como educación en deberes y derechos, para fortalecer su empoderamiento frente al sistema de salud.

En todos los grupos focales realizados, se presentaron participantes que instauraron acciones legales. Se resalta el caso de una paciente que reportó la ineficacia de las instancias jurídicas para conseguir que se le diera el medicamento, ya que, posterior a la tutela y desacato, se solicitó la detención del representante legal de la EPS y, aun así, a la fecha de cierre del presente estudio, se conoció que la paciente continuaba con dificultad para que le fuera entregado este. Dicha situación, junto a otras narrativas identificadas, expuso la percepción colectiva relacionada con la pérdida de poder de la acción de tutela para acceder a los servicios de salud.

Otro aspecto importante que se identificó dentro de los grupos focales fueron las experiencias sobre el gasto de bolsillo en los pacientes y sus familias. Que el promedio de gastos $(\$ 300.000)$ exprese la mitad del SMLMV de los colombianos, unido a las características de la población con MM (adulto mayor económicamente inactivo por la discapacidad y/o incapacidad del diagnóstico), junto a las barreras de acceso geográficas expuestas en el segmento de resultados, hace que se conviertan en factores de riesgo para la adherencia al tratamiento, deslegitimando los objetivos de oportunidad y calidad señalados por el SOGC ${ }^{4,6}$ y en el modelo de cuidado del paciente con cáncer 5 .

\section{Limitaciones}

Se desconoce el número de pacientes con mieloma múltiple en Colombia a 2017, lo que no permite especificar la representatividad estadística de la muestra utilizada. Por otro lado, dentro de los grupos focales, el número de participantes no responde a una muestra probabilística, por lo que no es posible señalar representatividad estadística de los casos, según región. 


\section{Referencias}

1. Instituto Nacional de Cancerología. Anuario estadístico 2013 [internet]. Disponible en: http://www.cancer.gov.co/files/libros/ archivos/anuarioestadistico2013

2. Globocan. Multiple myeloma - Estimated incidence and prevalence, adult population: both sexes [internet]. International Agency for Research on Cancer-World Health Organization; 2012. Disponible en: http://globocan.iarc.fr/old/ summary_table_site_prev.asp?selection $=17270 \&$ title $=$ Mult iple+myeloma\&sex $=0 \&$ america $=2 \&$ window $=1 \&$ sort $=0 \&$ sub mit=\% C2\%A0Execute

3. Kariyawasan CC, Hughes DA, Jayatillake MM, Mehta AB. Multiple myeloma: causes and consequences of delay in diagnosis. QJM. 2007;100(10):635-40.

4. Colombia, Ministerio de la Protección Social. Decreto 1011 de 2006. Sistema Obligatorio de Garantía de la Calidad de la Atención de Salud del Sistema General de Seguridad Social en Salud [internet]. [cited 2017 may 10]. Disponible en: http://www.alcaldiabogota.gov.co/sisjur/normas/Norma1. jsp?i=19975

5. Modelo de cuidado del paciente con cáncer. Serie de Documentos Técnicos Instituto Nacional de Cancerología No 5 [internet].
Bogotá, D.C.; 2015. Disponible en: http://www.cancer.gov.co/ files/libros/archivos/Modelo-para-el-control.pdf

6. Colombia, Ministerio de Salud y Protección Social. Resolución 1477 de 2016, "por la cual se define el procedimiento, los estándares y criterios para la habilitación de unidades funcionales para la atención integral de cáncer del adulto (UFCA) y de las unidades de atención de cáncer infantil (Uacai) [internet]. Disponible en: https://www.minsalud.gov.co/Normatividad_Nuevo/Resoluci\%C3\%B3n\%201477\%20de\%202016.pdf

7. Colombia, Ministerio de Salud. Resolución 8430 de 1993, "por la cual se establecen las normas científicas, técnicas y administrativas para la investigación en salud" [internet]. Disponible en: https://www.minsalud.gov.co/sites/rid/Lists/BibliotecaDigital/RIDE/DE/DIJ/RESOLUCION-8430-DE-1993.PDF

8. Abrahamsen B, Andersen I, Christensen SS, Skov Madsen J, Brixen K. Utility of testing for monoclonal bands in serum of patients with suspected osteoporosis: retrospective, cross sectional study. BMJ. 2005;330(7495):818.

9. Asociación Española de Afectados por Linfoma, Mieloma y Leucemia (AEAL). Informe 2015 de necesidades de los pacientes con mieloma múltiple y sus familiares en España. 